\title{
Investitionsfinanzierung und ineffiziente Krankenhausstrukturen
}

Christopher Hermann und Nadia Mussa

12.1 Ausgangslage - 232

12.1.1 Deutschland investiert zu wenig in Krankenhäuser - 233

12.1.2 GKV-Gesundheitsreform 2000 - großes Vorhaben mündet in mutlosem Stückwerk - 233

12.1.3 Fehlentwicklungen in der Krankenhausversorgung - 235

12.2 Zielbild Krankenhausversorgung - 236

12.3 Bundeseinheitliche Qualitäts- und Strukturvorgaben zielgerichtet aufeinander abstimmen - 237

12.4 Investitionsfinanzierung - $\mathbf{2 3 8}$

12.4.1 Neuordnung der Investitionsfinanzierung in einem systemischen Ansatz - 238

12.4.2 Neue Einzelförderung - 239

12.4.3 Leistungsorientierte Investitionsförderung - 239

12.4.4 Fortführung des Krankenhausstrukturfonds - 240

12.4.5 Digitalisierungsoffensive - 241

$12.5 \quad$ Fazit -241

$$
\text { Literatur - } 241
$$




\section{- - Zusammenfassung}

Im Juni 1999 verabschiedeten die damaligen Koalitionsfraktionen SPD und BÜNDNIS 90/DIE GRÜNEN einen Gesetzentwurf, der die Krankenhausversorgung umfassend reformieren sollte. Endlich sollte der über Jahrzehnte diskutierte Strukturwandel eingeläutet werden. Vom Gesetzesbeschluss wurden im Laufe des weiteren Verfahrens nur Teile umgesetzt. Seitdem folgten unzählige Änderungen, die eine Fülle von Detailregelungen enthielten. Von der ursprünglichen Vision - Akteure der Krankenhausversorgung sollen auf Augenhöhe den Krankenhausmarkt bedarfsgerecht und qualitätsorientiert gestalten - blieb jedoch wenig übrig. Viele aktuell diskutierte Probleme, wie Fehlentwicklungen in der Krankenhausversorgung, chronische Unterfinanzierung der Investitionsbedarfe und ineffiziente Krankenhausstrukturen, ergeben sich aus dem fehlenden Mut der politischen Verantwortungsträger, eine Gesamtkonzeption und Vision der Krankenhausversorgung der Zukunft auf den Weg zu bringen. Deutschland investiert zu wenig in die Krankenhausinfrastruktur. Während die hausgemachten Probleme weiterhin nicht gelöst sind, ergeben sich aufgrund demografischer Entwicklungen, medizinisch-technischen Fortschritts, ökologischer Herausforderungen und den Möglichkeiten von Künstlicher Intelligenz und molekularer Medizin ganz neue Herausforderungen für das Gesundheitswesen. Es ist an der Zeit, die vielen vorhandenen Ideen und Regulierungsvorschriften in ein stimmiges Gesamtbild zu überführen und den Investitionsbedarf der Krankenhäuser anhand eines Zielbilds der Krankenhausversorgung der Zukunft sachgerecht zu ermitteln und bedarfsgerecht $z u$ finanzieren. Auf diese Weise sollte der Strukturwandel gelingen und Patientinnen und Patienten im Krankenhaus durchgehend mit angemessener Qualität und Effizienz behandelt werden können.

In 1999, a fundamental reform of the hospital market in Germany was planned. After decades of discussing inefficient hospital structures, a complete reorganisation of financing and responsibilities was finally supposed to solve the problems. In the end, due to a lack of courage and assumed loss of power in the regions, only parts of the reform could be pushed through the legislative process. Since then, countless changes followed, but only details were regulated, the "big picture" still hasn't been painted. Little remained of the original vision where players in hospital care should create together a hospital market that would ensure quality and need-based hospitals. Many currently discussed problems, such as underfinanced hospitals and inefficient structures, are due to a policy that fails to acknowledge that hospital treatment is imbedded in a complex system. Germany invests too little into its hospital infrastructure. While selfinflicted problems are still not solved, there are new challenges ahead such as demographic development, medical-technical progress, artificial intelligence, ecological challenges and molecular medicine. It is about time to restructure and reorganize the many existing ideas and regulations and to create a consistent concept. The appropriate amount of investment needs must be calculated and financed adequately. In this way, structural change and appropriate treatment quality and efficiency can be achieved.

\subsection{Ausgangslage}

Die Krankenhausversorgung ist eingebettet in ein System komplexer Wirkzusammenhänge, dennoch wird die Krankenhausfinanzierung in der politischen Wahrnehmung nicht systemisch betrachtet. Jede Herausforderung wird in hoher Detaillierung mit Hilfe von mechanistischen Einzellösungen durchreguliert - im Ergebnis heben sich die gewünschten Effekte häufig gegenseitig auf. Mangelnder struktureller politischer Gestaltungswille und übertriebener Sparzwang haben zu Über-, Unter- und Fehlversorgung in Krankenhäusern geführt. 


\subsubsection{Deutschland investiert zu wenig in Krankenhäuser}

Der Befund ist eindeutig: Krankenhausstrukturen werden seit Jahrzehnten unterfinanziert. Die Höhe des Investitionsbedarfs hängt von Bestand, Bausubstanz und dem Entwicklungsziel für eine Krankenhausversorgung der $\mathrm{Zu}-$ kunft ab. Ein Zielbild für optimale Krankenhausstrukturen existiert in Deutschland nicht. Wie hoch der Investitionsbedarf in Krankenhäusern tatsächlich ist, kann deshalb nicht genau beziffert werden. Das RWI - Leibniz-Institut für Wirtschaftsforschung schätzte 2017 den jährlichen Bedarf auf ca. 5,4 Mrd. $€$ allein für den Erhalt der bestehenden Krankenhausbausubstanz (Augurzky et al. 2017). In einer aktuellen gemeinsamen Verlautbarung der Deutschen Krankenhausgesellschaft (DKG), des Spitzenverbandes der Gesetzlichen Krankenversicherung (GKV-SV) und des Verbandes der Privaten Krankenversicherung (PKV) wird der jährliche Investitionsbedarf auf weit über $6 \mathrm{Mrd} € €$ veranschlagt (DKG et al. 2019).

Die Länder sind gesetzlich für die Finanzierung der Investitionen zuständig. Im Jahr 2017 haben sie nominal 2,76 Mrd. € an Fördermitteln zur Verfügung gestellt (DKG 2018). In den Jahren seit 1991 haben die Bundesländer in ihrer Gesamtheit stetig zu wenig Investitionsmittel bereitgestellt. Die DKG kommt zu dem Schluss, dass im Jahr 2017 real nur noch 50 \% der im Jahr 1991 finanzierten Summe aufgebracht wurde. Seit Jahrzehnten werden den Krankenhäusern nur knapp die Hälfte der benötigten Mittel zugeführt.

Bis zum Jahr 1972 finanzierten die Krankenkassen Behandlungsaufwände und Investitionsbedarfe der Krankenhäuser aus einer Hand. Die Defizite der Krankenhäuser waren immer weiter gestiegen, sodass schließlich die Bundesregierung im Mai 1969 einen Bericht über die finanzielle Lage der Krankenanstalten vorlegte, aus dem hervorging, dass das Defizit im Jahr 1966 über 840 Mio. DM betrug und für die Folgejahre mit einem weite- ren Anstieg des Defizits zu rechnen war (Bericht der Bundesregierung 1966). Festgestellt wurde ebenfalls, dass die Defizite der Krankenhäuser in unterschiedlichem Umfang teilweise von den Ländern ausgeglichen wurden. Daraus leitete die Bundesregierung ab, dass die Länder auch künftig bereit wären, sich an der Finanzierung der Krankenhausversorgung zu beteiligen. Über eine Grundgesetzänderung wurde die duale Finanzierung etabliert. Der Bund erhielt im Rahmen der konkurrierenden Gesetzgebung die Möglichkeit, die Krankenhausbetriebsmittelfinanzierung zu gestalten. Die Krankenhäuser erhalten seitdem Finanzmittel von den Krankenkassen, um ihre Betriebskosten zu decken, und von den Ländern, um ihren Investitionsbedarf zu finanzieren.

In der Folge kamen die Länder ihrer Finanzierungsverpflichtung weiterhin sehr unterschiedlich und insgesamt immer zurückhaltender nach. Wie ausgeführt wurden die Finanzmittel real von Jahr zu Jahr reduziert. Zahlreiche Gesetzesänderungen begleiteten diese Entwicklung, eine umfassende und zunehmend dringend benötigte Neugestaltung der gesetzlichen Rahmenbedingungen blieb jedoch bisher aus.

\subsubsection{GKV-Gesundheitsreform 2000 - großes Vorhaben mündet in mutlosem Stückwerk}

Die Krankenhausstrukturen in Deutschland sind historisch gewachsen. Eine gezielte und konkrete Weiterentwicklung der Strukturen war und ist nicht in Sicht. Zuletzt versuchte die damalige Koalition im Jahr 2000 durch die Einführung eines fallpauschalierten Vergütungssystems (DRG-System), Krankenhausversorgung umfassend neu zu gestalten. Man ging davon aus, dass die Krankenhausstrukturen ineffizient seien und einer Veränderung bedürften. Mit dieser Gesundheitsreform wurde u. a. das Ziel verfolgt, die Leistungsstruktu- 
ren im Krankenhaus zu verändern. Es sollte zu Schwerpunktbildungen, dem Abbau von Überkapazitäten und verstärkter Kooperation von Krankenhäusern kommen (Tuschen und Trefz 2010). Während der ursprüngliche Gesetzesentwurf zur "GKV-Gesundheitsreform 2000“ ein umfassendes Versorgungs- und Finanzierungskonzept beinhaltete (Deutscher Bundestag 1999; Hermann 2007) und damit echte Chancen für eine Modernisierung und kontinuierliche Weiterentwicklung der Krankenhausversorgung bot, wurde am Ende des Gesetzgebungsverfahrens nur ein Teil der Pläne umgesetzt.

Viele Ideen und Impulse, die mit der GKVGesundheitsreform 2000 ursprünglich verbunden waren, sind auch heute weiterhin nachvollziehbar. Dem Gesetzentwurf ist zu entnehmen, dass mit Einführung einer fallpauschalierten Vergütung flankierend geplant war, schrittweise eine monistische Finanzierung einzuführen. Im ersten Schritt sollten die zunächst nur vorübergehend durch die Krankenkassen zu finanzierenden Instandhaltungsaufwände dauerhaft in die Pflegesätze integriert werden. In einem zweiten Schritt sollten die pauschalen Fördermittel, die der Wiederbeschaffung kurzfristiger Anlagegüter sowie kleiner baulicher Maßnahmen dienen, in die Fallpauschalen integriert werden. Hierzu sollten die bisher durch die Länder finanzierten Mittel an die Krankenkassen weitergeleitet werden, damit diese die Mittel über die Fallpauschalen hätten ausschütten können. In einem dritten Schritt hätte schließlich auch die Einzelförderung mittels der Betriebserlöse auf die Finanzierung übergehen sollen. Im Zuge der Reform war geplant, den Krankenkassen wesentlich mehr Mitgestaltungsmöglichkeiten und Entscheidungskompetenzen bei der Krankenhausstrukturentwicklung einzuräumen.

Während die Finanzierung der Instandhaltungspauschale tatsächlich dauerhaft in die Pflegesatzfinanzierung überführt wurde, blieben die Schritte 2 und 3 aus. Auch Mitwirkungsrechte für Krankenkassen sind in der geplanten Form nicht eingeführt worden. Dadurch entstand ein Gestaltungsva- kuum. Den Krankenkassen fehlen einerseits Durchsetzungsrechte, um als verlässlicher Vertragspartner Qualität in der Krankenversorgung voranzubringen. Viele Länder kommen andererseits ihrer Verantwortung nur unzureichend nach.

Mit dem Krankenhausfinanzierungsreformgesetz (KHRG) wurde das Thema Investitionsfinanzierung im Jahr 2009 erneut angegangen. Investitionsförderung sollte durch leistungsorientierte Investitionspauschalen ermöglicht werden. Den Ländern wurde freigestellt, ob sie bei ihrer herkömmlichen Art der Pauschal- und Einzelförderung bleiben oder auf die leistungsorientierten Investitionspauschalen umsteigen wollten. Seit dem Jahr 2014 wird jährlich vom Institut für Entgeltkalkulation (InEK) ein Katalog mit Investitionsbewertungsrelationen (IBR) vorgelegt. Investitionsmittel könnten bei Anwendung je nach kalkuliertem Investitionsaufwand zur Erbringung bestimmter Leistungen bedarfsgerecht erlöst werden. So entstehen für strahlentherapeutische Maßnahmen andere Investitionsaufwände als etwa bei der Behandlung von Verletzungen an Schulter, Knie oder Handgelenk. Investitionsmittel können durch IBR sachgerechter ausgezahlt werden als durch eine pauschale Auszahlung pro Bett, wie weiterhin überwiegend durch die Länder praktiziert wird.

2016 schließlich wurde im Rahmen des Krankenhausstrukturgesetzes (KHSG) der Krankenhausstrukturfonds eingeführt. Die gesetzlichen Krankenkassen wurden an der Finanzierung von Investitionsmaßnahmen zur Verbesserung von Versorgungsstrukturen beteiligt und erhielten Mitbestimmungsrechte zum Einsatz der Mittel; die Länder mussten sich hälftig an der Finanzierung dieser Maßnahmen beteiligen und durften gleichzeitig ein bestimmtes Investitionsförderniveau der letzten Jahre für die sonstige Einzel- und Pauschalförderung nicht unterschreiten. Der Krankenhausstrukturfonds wurde mit dem Ziel, die Krankenhausstrukturen zu verbessern, etabliert und zeitlich eng begrenzt. Mit dem Pflegepersonalstärkungsgesetz (PpSG) wurde 
der Krankenhausstrukturfonds für die Jahre 2019 bis 2022 fortgeführt und umfasst insgesamt über beide Programme bis zu $5 \mathrm{Mrd}$. an zusätzlichen Mitteln, um die Krankenhausstrukturen zu verändern. ${ }^{1}$

Seit Einführung der DRGs hat der Gesetzgeber das fallpauschalierte Vergütungssystem in ein detailliertes und mittlerweile überreguliertes Finanzierungssystem fortgeschrieben. Mit dem PpSG wurde im letzten Jahr zudem mit der Ausgliederung der Pflegepersonalaufwendungen die Rückentwicklung in ein teilweise selbstkostendeckungsorientiertes System eingeleitet.

Das DRG-System entfaltete den gewünschten Kostendruck auf die Krankenhäuser, um Betriebsabläufe wirtschaftlicher $\mathrm{zu}$ gestalten. Ohne ausreichende Investitionen in Strukturen konnten Wirtschaftlichkeitsreserven jedoch nicht ohne Auswirkungen auf die Qualität der Behandlung realisiert werden. Vor diesem Hintergrund reagierte der Gesetzgeber seit dem Ende der Konvergenzphase 2009 immer wieder mit Eingriffen, um den Krankenhäusern zu Lasten der Beitragszahler zusätzliche Finanzmittel zur Verfügung zu stellen.

Im Ergebnis geht ein wesentlicher Fehlanreiz des letzten Jahrhunderts seit Einführung des DRG-Systems vom Festhalten an der dualen Finanzierung bei gleichzeitiger realer Reduzierung der Investitionskostenübernahme durch die Länder aus: Krankenhäuser, die (in ihren Augen) keine ausreichenden Investitionsmittel erhielten, sahen sich dazu gezwungen, Betriebserlöse aus den Fallpauschalen für Investitionsmaßnahmen zu verwenden.

\subsubsection{Fehlentwicklungen in der Krankenhausversorgung}

Krankenhäuser geben pro Jahr ca. 6 Mrd. € für Investitionen aus (DKG et al. 2019) und setzen aufgrund der unzureichenden Finanzaus-

Nähere Informationen zum Krankenhausstrukturfonds finden sich auch im Beitrag von Augurzky et al., $>$ Kap. 17 in diesem Buch. stattung durch die Länder Erlöse aus Behandlungen zweckentfremdet in erheblicher Höhe hierfür ein. Das DRG-System ist transparent gestaltet. Jedes Krankenhaus kann anhand des eigenen Leistungsportfolios ermitteln, für welche Leistungen es positive Deckungsbeiträge generieren kann. Das Institut für das Entgeltsystem im Krankenhaus (InEK) kalkuliert und veröffentlicht in jedem Jahr, welche Aufwände einzelnen Fallpauschalen zugrunde gelegt wurden. So ist ersichtlich, welche Aufwände z. B. für Implantate, ärztliche Kosten, Pflegeaufwände etc. in die Kalkulation eingeflossen sind (InEK 2019a).

Liegen der Kalkulation für die Implantation von Hüftgelenks-Totalendoprothesen (HüftTEPs) beispielsweise sehr hohe Implantatekosten zugrunde, könnte das Krankenhaus ein positives Ergebnis erzielen, indem es günstigere Implantate einkauft - z. B. billige Plastikware etwa aus Fernost statt hochwertiger Keramik. Wer mehr Fälle behandelt, ohne in gleichem Maße mehr Personal zu beschäftigen, kann ebenfalls Deckungsbeiträge zur Finanzierung von Investitionen erwirtschaften. Je höher der ökonomische Druck auf einem Krankenhaus lastet, umso schmaler wird der Grad zwischen sinnvoller Effizienzsteigerung und qualitätsgefährdendem Sparen.

Anhand weniger Beispiele wird bereits deutlich, dass dieser Grad inzwischen häufig verlassen wird.

- Beispiel Pflege: Krankenhäuser haben bei der Personalausstattung ihren Fokus insbesondere auf das ärztliche Personal und den Funktionsdienst (z. B. Pflegekräfte im OPSaal) gelegt. Gleichzeitig wurde das Personal in den bettenführenden Abteilungen so weit reduziert, dass der Gesetzgeber meinte, einem weiteren Abbau mit Pflegepersonalfördermitteln entgegenwirken zu müssen und nunmehr - wenig durchdacht - die Pflegekosten aus dem DRG-System ausgliedern lässt.

- Beispiel Fallzahlsteigerung: Die Zahl der Behandlungsfälle je 100.000 Einwohner ist bis zum Jahr 2017 im Vergleich zu 2000 um $15,4 \%$ gestiegen. Durch demografischen 
Wandel und Morbiditätsentwicklung der Bevölkerung allein können solche Fallzahlsteigerungen nicht schlüssig erklärt werden (Schelhase 2019).

- Beispiel Hüft-TEPs: Im Jahr 2014 wurden in Deutschland an 1.090 Krankenhäusern künstliche Hüftgelenke eingesetzt, also quasi an jedem zweiten Krankenhaus im Land (Mansky et al. 2018). Für diesen Leistungsbereich wurde inzwischen eine gezielte $\mathrm{Ab}$ wertung der Fallpauschale vorgenommen, um keine weiteren ökonomischen Anreize zur Fallzahlausweitung zu setzen.

Vielen Fehlentwicklungen hätten die Länder aufgrund ihrer weitreichenden Kompetenzen in der Krankenhausplanung entgegenwirken können. In den 1990er und 2000er Jahren haben sie sich jedoch vielfach von einer konkreten Krankenhausplanung verabschiedet und darauf beschränkt, in hohem Umfang Rahmenvorgaben zu formulieren. Eine in die Zukunft gerichtete (Rahmen-)Bedarfsplanung ist meist nicht erkennbar. Die Krankenhausträger entscheiden somit in hohem Maße selbst, welche konkreten Leistungen sie anbieten wollen.

Im Ergebnis haben sich ineffiziente Krankenhauskapazitäten verfestigt. Hüft-TEPs werden beispielsweise nicht nur an zu vielen Standorten eingesetzt, sondern vielen Krankenhäusern fehlt aufgrund geringer jährlicher Fallzahlen auch die Erfahrung, um eine qualitativ hochwertige Versorgung kontinuierlich sicherzustellen. Im Jahr 2014 haben 262 Krankenhäuser die Leistung weniger als 43-mal erbracht (Mansky et al. 2018). Legt man die Maßstäbe der Deutschen Gesellschaft für Orthopädie und orthopädische Chirurgie zugrunde, muss ein Endoprothetik-Zentrum der Maximalversorgung mindestens 200 Implantationen pro Jahr erbringen, davon mindestens 50 Hüftimplantationen, um zertifiziert zu werden (endoCert 2018). Die Vielzahl der Standorte mit teilweise deutlich zu niedrigen Fallzahlen führt zudem dazu, dass insgesamt mehr Fachkräfte benötigt werden als bei einer rationaleren Verteilung der Fälle auf weniger Krankenhäuser.
Ohne Mindestfallzahlen in wichtigen Leistungsbereichen und eine konkrete Zuweisung von Versorgungsaufträgen wird sich auch an der zu hohen Zahl kleiner Krankenhäuser in Deutschland nicht so schnell etwas ändern. Im Jahr 2015 wiesen $39 \%$ der bettenführenden Akutkrankenhäuser weniger als 150 Betten aus. Sie schneiden bereits aufgrund ihrer Größe wirtschaftlich schlechter ab als größere Kliniken; was auf die im Vergleich höheren Vorhaltekosten zurückgeführt wird (Augurzky et al. 2018). Krankenhausschließungen sind nicht populär; häufig werden unprofitable und auch unter Qualitätsaspekten entbehrliche Krankenhäuser zu lange betrieben und mit kommunalen Finanzspritzen Schließungen zumindest hinausgezögert.

\subsection{Zielbild Krankenhausversorgung}

Das Krankenhauswesen bedarf einer klaren Orientierung, wohin es sich perspektivisch entwickeln soll. Die Qualität der Patientenversorgung muss in den Mittelpunkt rücken und zukunftsfeste Strukturen müssen daran ausgerichtet werden. Krankenhausstrukturen sind höchstkomplex und können nicht kurzfristig flexibel angepasst werden. Die Länder müssen für längere Planungszeiträume prospektiv kapazitätsorientierte Vorstellungen davon entwickeln, wo welche Bedarfe bestehen und wo sie sich absehbar ergeben werden.

Diese Notwendigkeit wurde in anderen europäischen Ländern schon viel früher erkannt und umgesetzt. In der Schweiz wurde inzwischen eingeführt, dass Krankenhauspläne auf einen Zeithorizont von zehn Jahren ausgerichtet und in einem rollierenden System alle drei Jahre angepasst werden, bevor die nächste große Planungsphase einer Dekade greift (Kanton Zürich Gesundheitsdirektion 2011). Dänemark hat 2007 eine große Krankenhausreform in Gang gesetzt. Der künftige Bedarf an Krankenhäusern und an Notfallversorgung wurde analysiert. Es wurden Empfehlungen ausgespro- 
chen, welche Krankenhausstrukturen in $\mathrm{Zu}$ kunft sinnvoll und notwendig wären. Daraus ergaben sich konkrete Anforderungen an Investitionsbedarfe und Veränderungen (Henriksen 2019).

In Deutschland wird von Experten ebenfalls mehr Gestaltungswille gefordert. Im Diskussionspapier der Leopoldina wurde 2016 die Politik dazu aufgefordert, endlich mit Mut notwendige Strukturänderungen anzugehen (Busse et al. 2016). Zuletzt wurde in einer Studie im Auftrag der Bertelsmann Stiftung aufgezeigt, wie durch eine gezielte Reduzierung von Krankenhausstandorten die Qualität und Effizienz der Krankenhausversorgung einer Region deutlich vorangebracht werden kann (Loos et al. 2019).

Die Länder haben durch ihre Planungsund Finanzierungsverantwortung gewichtige Instrumente in der Hand, mit deren Hilfe sie Maßstäbe in der Gesundheitsversorgung setzen können. Neben der Gestaltung von Strukturen bietet sich bei den ca. 2.000 Krankenhäusern in Deutschland auch ein enormes Kapital, um nachhaltiges Bauen gezielt zu fördern und im Zeichen des Klimawandels ein positives Exempel für ökologisches und klimaneutrales Bauen zu setzen. Auch dieser Aspekt bleibt bisher meist einzelnen Krankenhäusern überantwortet - ein Beleg dafür, dass politisch zu wenig integriert gedacht wird und die bestehenden Gestaltungsoptionen nicht konsequent genutzt werden.

In der Tat: Es ist höchste Zeit, dass in Deutschland die Weichen auf Zukunft gestellt werden.

\subsection{Bundeseinheitliche Qualitäts- und Strukturvorgaben zielgerichtet aufeinander abstimmen}

Bundeseinheitliche Qualitäts- und Strukturvorgaben sind ein wichtiges Instrument, um Patientensicherheit in den Mittelpunkt zu rücken. Sie sollten Versorgung rahmen und ver- hindern, dass ökonomische Anreize zu Lasten der gebotenen Qualität Vorrang erhalten. Qualitätsrichtlinien müssen deshalb verbindlich durchsetzbar sein und Impulse für die Entwicklung von Zielbildern der Krankenhausversorgung der Zukunft setzen.

Instrumente zur Gestaltung und Regulierung der Krankenhausversorgung wurden in den letzten Jahrzehnten zahlreich geschaffen. Insbesondere die Behandlungsqualität ist immer mehr in den Vordergrund gerückt - nicht zuletzt als Reaktion auf die aufgezeigten Fehlentwicklungen.

Wer krank ist, hat überall in Deutschland Anspruch auf die notwendige medizinische Versorgung, die dem anerkannten Stand der medizinischen Erkenntnisse entsprechen muss. Evidenzbasierte Medizin und Strukturvorgaben für die Durchführung von Behandlungsverfahren und Therapien sind wichtige Elemente, um notwendige Qualitätsstandards zu gewährleisten.

Viele sinnvolle Maßnahmen zur Verbesserung der Qualität in der Krankenhausversorgung bedürfen einer Ordnung und Priorisierung, um ihre Wirkung besser zu entfalten. Insbesondere wurde der Gemeinsame Bundesausschuss (G-BA) eingebunden, dessen Verantwortungsbereich zur Weitentwicklung der Qualität inzwischen umfassend ausgestaltet ist:

- Der G-BA legt insbesondere für aufwendige medizintechnische Leistungen u.a. Mindestanforderungen an die Struktur-, Prozess- und Ergebnisqualität fest.

- Der G-BA legt Maßnahmen zur Sicherung der Hygiene in der Versorgung fest.

- Der G-BA bestimmt verbindliche Mindestvorgaben für die Ausstattung der stationären Einrichtungen in der psychiatrischen und psychosomatischen Versorgung mit therapeutischem Personal.

- Der G-BA legt Mindestmengen fest für planbare Leistungen, bei denen die Qualität des Behandlungsergebnisses von der Menge der erbrachten Leistungen abhängig ist.

- Der G-BA beschließt Qualitätsindikatoren zur Struktur-, Prozess- und Ergebnisqua- 
lität als Grundlage für qualitätsorientierte Entscheidungen der Krankenhausplanung.

- Der G-BA beschließt bundeseinheitliche Vorgaben für die Vereinbarung von Sicherstellungszuschlägen (insbesondere ist festzulegen, in welcher Entfernung welche Leistungen erreichbar sein müssen, um die Versorgung der Bevölkerung sicherzustellen).

- Der G-BA legt ein gestuftes System von Notfallstrukturen in Krankenhäusern fest.

- Der G-BA konkretisiert die besonderen Aufgaben von Zentren und Schwerpunkten.

- Der G-BA legt Pflegepersonaluntergrenzen in pflegesensitiven Bereichen der Krankenhäuser fest.

Aus der umfänglichen Regelungskompetenz des G-BA erwachsen eine Fülle an Vorgaben, die für die beteiligten Akteure in den Ländern jedoch keine strukturierte Planungsgrundlage ergeben, sodass heute getroffene Entscheidungen bereits morgen hinfällig werden können. Häufig sind einzelne Aufgaben des G-BA als Reaktion der Politik auf aktuelle Problemlagen entstanden, wie z. B. die Vorgabe, ein gestuftes System von Notfallstrukturen festlegen oder Pflegepersonaluntergrenzen in pflegesensitiven Bereichen definieren zu lassen. Es fehlt ein Gesamtkonzept, welche Qualitätsvorgaben langfristig und dauerhaft auf Bundesebene benötigt werden, mit welcher Zielrichtung diese gestaltet werden sollen und in welcher Priorisierung bestimmte Themen anzugehen sind. Das Aufgabenportfolio wäre konsequent auf Qualität und Patientensicherheit auszurichten. Steuerungselemente, wie z. B. die Definition von besonderen Aufgaben von Zentren und Schwerpunkten, greifen zu sehr in die Planungskompetenz der Länder ein, ohne die Versorgungsqualität entscheidend voranzubringen. Zuständigkeiten müssen sich sinnvoll ergänzen und dürfen nicht in Konkurrenz zueinander gesetzt werden, wie es exemplarisch bei der Zentrenpolitik mittlerweile der Fall ist.

Bevor noch weitere Aufgaben auf den GBA übertragen werden, wäre sicherzustellen, dass diese überhaupt in einem überschaubaren
Zeitrahmen und in der gebotenen Qualität koordiniert abgearbeitet werden können.

\subsection{Investitionsfinanzierung}

\subsubsection{Neuordnung der Investitionsfinanzierung in einem systemischen Ansatz}

Die Investitionsfinanzierung bleibt ein zentrales Instrument zur Gestaltung von Krankenhausversorgung. Dabei muss die Investitionsfinanzierung in eine übergeordnete Gesamtkonzeption der Krankenhausversorgung eingebettet sein. Eine Neuordnung beinhaltet folgende Aspekte:

- Die wesentlichen Akteure - Krankenhäuser, GKV und Länder - werden mit Kompetenzen ausgestattet, die einen Ausgleich der Interessen zum Wohle der Patienten herbeiführen.

- In den Ländern entwickelte Zielbilder künftiger Versorgungsstrukturen sind Grundlage für Investitionsentscheidungen.

- Investitionsfinanzierung wird zur Entwicklung und Steuerung von Krankenhausstrukturen eingesetzt.

- Nachhaltiges und klimaneutrales Bauen wird Fördervoraussetzung.

- Bauplanung führt zu Prozessoptimierung von Behandlungsabläufen.

- Vorhandene Instrumente zur Finanzierung von Investitionen werden aufeinander abgestimmt; Bauvorhaben sind verlässlich und zügig umsetzbar.

- Hemmnisse, die effizientem Bauen im Wege stehen, werden ausgeräumt.

- Herausforderungen, die unmittelbar und umfassend angegangen werden müssen, wie Strukturwandel und Digitalisierung, werden durch Sonderfinanzierungsprogramme bewältigt.

Insgesamt besteht ein Mix an verschiedenen Investitionsfinanzierungsinstrumenten, der grundsätzlich Möglichkeiten für eine 
sinnvolle und zielgerichtete Investitionsfinanzierung enthält. Allerdings gilt es, die verschiedenen Instrumente zunächst zu schärfen, neu auszurichten und anschließend auch kraftvoll voranzubringen. Wesentliche Elemente der neuen Investitionsfinanzierung sind Einzelförderung, Pauschalförderung und Sonderfinanzierungsprogramme.

\subsubsection{Neue Einzelförderung}

Krankenhausneubauten und wesentliche Erweiterungsbauten müssen richtungsweisende Elemente zur Perspektivensetzung und Neuordnung von Strukturen sein und als Vorbild für die Krankenhausversorgung künftiger Zeiträume dienen. Bestandskrankenhäuser müssen in vorgegebenen Zeiträumen in Richtung solcher Vorzeigeobjekte weiterentwickelt werden. So kann eine fortgesetzte Spirale entstehen, die für Sanierungs- und Modernisierungsbedarfe richtungsgebend ist und immer wieder durch Neuentwicklungen positiv angereizt wird. Die Länder sollen im Rahmen der dualen Finanzierung die Einzelförderung solcher Baumaßnahmen übernehmen.

Der Rechtsrahmen ist weiterzuentwickeln, um Investitionsfinanzierung in ausreichender Höhe und zielgerichtet $\mathrm{zu}$ ermöglichen und von kurzfristigen politischen Interessenlagen zu entkoppeln. Die Länder sind zur Aufstellung von Rahmenplänen zu verpflichten, die ein konkretes Zielbild für die Versorgungsstrukturen künftiger Zeiträume beinhalten. Die GKV ist in die Erarbeitung dieses Zielbildes einzubinden. Geeignete Konfliktlösungsmechanismen, wie die Durchführung von Schlichtungsverfahren, sind einzuführen, um jederzeit eine umfassende sachliche Auseinandersetzung mit der Materie zu gewährleisten. Weitere wichtige Akteure in den Regionen sind bei der Entscheidungsfindung anzuhören und Einvernehmen ist anzustreben. Regionale Besonderheiten sind $\mathrm{zu}$ berücksichtigen, um passgenaue Lösungen für die Menschen vor Ort zu gewährleisten.
Investitionsbedarfe zur Umsetzung der Zielbildstrukturen sind landesindividuell und zukunftsgerichtet zu beziffern. Die gesetzlichen Grundlagen müssen angepasst werden, um die Umsetzung der identifizierten Bedarfe rechtlich durchsetzbar $\mathrm{zu}$ gestalten. Eine zielgenaue Verwendung der Finanzierungsmittel bedarf einer klaren Priorisierung zwischen grundgesetzlich verankerter Berufsfreiheit und Patientensicherheit. Viele Länder haben sich in der Vergangenheit aus einer umfassenden Krankenhausplanung zurückgezogen, da ihre Entscheidungen u.a. vor den Gerichten immer wieder an Artikel 12 GG abgeprüft wurden und der Berufsfreiheit (und damit wirtschaftlichen Interessen) rechtlich ein höheres Gewicht zugesprochen wurde als an guter Versorgungsqualität ausgerichteten krankenhausplanerischen Entscheidungen. Krankenhausplanerische Auswahlentscheidungen sind konsequent an Qualitätskriterien auszurichten und entsprechend vorrangig $\mathrm{zu}$ bewerten.

Die bestehende Form der Einzelförderung ist auf neue Grundlagen zu stellen. Die Fülle an Baurichtlinien und Vorschriften ist intransparent und steht einer wirtschaftlichen Bauweise entgegen. Bauvorhaben müssen in wesentlich kürzeren Zeiträumen verlässlich planbar und umsetzbar werden. Für die Einzelförderung ist in den Ländern ein Kriterienkatalog zu veröffentlichen, welche Baumaßnahmen grundsätzlich förderfähig und welche Kriterien bereits bei der Bauplanung zu beachten sind. Es sind Rahmenvorgaben für das Krankenhaus der Zukunft zu erstellen, die absehbare Versorgungstrends aufgreifen und deren Umsetzung zur Fördervoraussetzung machen.

\subsubsection{Leistungsorientierte Investitionsförderung}

Es bedarf einer vollständigen Umstellung der Investitionsfinanzierung von Instandhaltungsmaßnahmen, der Wiederbeschaffung kurzfristiger Anlagegüter sowie kleiner baulicher Maß- 
nahmen auf leistungsorientierte Investitionsbewertungsrelationen. Das InEK hat seit dem Jahr 2014 Erfahrungen darin gewonnen, wie die tatsächliche Investitionstätigkeit der Krankenhäuser mit verschiedenen Leistungsangeboten zusammenhängt. Die Investitionsbedarfe unterscheiden sich zwischen verschiedenen Leistungen teilweise um das Zehnfache (InEK 2019b). Diesen Unterschieden sollte Rechnung getragen und den Krankenhäusern zielgerichtet ausreichend Mittel zur Verfügung gestellt werden, damit sie unbürokratisch die notwendige Finanzierung erlösen können und aufwändige Antragsverfahren entfallen. Die Krankenhausträger sollten einen Jahresbericht vorlegen, in welcher Höhe Investitionsmittel erlöst und für welche Maßnahmen diese eingesetzt wurden. So wäre gewährleistet, dass die notwendigen Instandhaltungs- und Modernisierungsmaßnahmen auch tatsächlich erfolgen.

Die Mittel für die leistungsorientierte Pauschalförderung sollten von den Ländern über den Gesundheitsfonds an die Krankenkassen ausgeschüttet werden. Dabei wäre sicherzustellen, dass vor der Überführung der Mittel eine Basiseinschätzung über den jährlichen Investitionsbedarf vorgenommen wird. Im Weiteren sollte über jährliche landesweite Verhandlungen der Basisbedarf zwischen Krankenkassen, Krankenhausgesellschaft und Land fortgeschrieben werden. Als Konfliktlösungsmechanismus bietet sich eine Schiedsstelle an, wie dies bereits im Entwurf zur GKV-Gesundheitsreform 2000 vorgesehen war. Gegebenenfalls sind die Krankenkassen an der Finanzierung dieser Investitionsmaßnahmen zu beteiligen. Auf diese Weise kann der schleichende Übergang der Investitionsfinanzierung von den Ländern auf die Krankenkassen aufgelöst und mit klaren Mitgestaltungs- und Finanzierungsverantwortungen verknüpft werden.

\subsubsection{Fortführung des Krankenhausstrukturfonds}

Der Krankenhausstrukturfonds ist grundsätzlich ein geeignetes Instrument, um den Abbau von kapazitätsbedingter Überversorgung voranzubringen und die Konzentration von aufwändigen und komplexen Leistungen zu fördern. Dazu ist er zweckentsprechend und mit ernsthaftem politisch gestalterischem Willen umzusetzen.

Derzeit stehen einer durchgreifenden und schnellen Weiterentwicklung der Strukturen häufig auch lokalpolitische Herausforderungen im Wege. Der Krankenhausstrukturfonds hat dazu geführt, dass notwendige und unvermeidliche Schließungen vor allem von sehr kleinen Krankenhausstandorten angegangen werden konnten. Insbesondere ist es sinnvoll und richtig, den Krankenhausstrukturwandel damit zu verbinden, vor Ort alternative medizinische Versorgungsangebote zur Absicherung der Versorgung der Menschen zu schaffen. Insofern ist bei der Formulierung eines Zielbildes über Krankenhausstrukturen der Zukunft unbedingt zu berücksichtigen, wie die Gesamtversorgung und der schnelle Zugang zu medizinischer Infrastruktur in den Regionen gewährleistet bleiben, wenn regional Krankenhausstandorte oder stationäre Versorgungsangebote wegfallen.

Da der Strukturwandel wesentlich langsamer einsetzte, als dies bei der Einführung der Fallpauschalen im Jahr 2003 erwartet wurde, besteht noch über mehrere Jahre der Bedarf, diesen Wandel gezielt mit zweckgebundenen zusätzlichen Fördermitteln zu unterstützen. Der Krankenhausstrukturfonds sollte konsequent ausschließlich zum Abbau von Überversorgung und der Konzentration von Leistungsangeboten genutzt werden. Fälschlicherweise hat der Gesetzgeber in der Weiterentwicklung des Krankenhausstrukturfonds bereits weitere Fördertatbestände aufgenommen, wie z. B. die Förderung von Vorhaben zur Verbesserung der informationstechnischen Sicherheit der Krankenhäuser. Lokalpolitisch 
ist es reizvoller, ein Krankenhaus mit zusätzlichen Fördermitteln auszustatten, die den Erhalt des Standortes unterstützen, als einen Standort zu schließen. Diese Sachverhalte dürfen allerdings nicht länger miteinander vermischt werden.

\subsubsection{Digitalisierungsoffensive}

Der Einsatz von neuer Informationstechnologie (IT) im deutschen Gesundheitswesen ist alles andere als optimal. Stephani et al. (2019) konnten zeigen, dass Deutschland im Vergleich zu anderen Ländern (darunter die Türkei und die USA) unterdurchschnittlich digitalisiert ist. Als Gründe hierfür werden u. a. mangelnde Investitionen ausgemacht.

Eine Digitalisierungsoffensive, ein Digital Boost, erweist sich als dringend notwendig. Hierbei sollte festgelegt werden, welcher Einsatz von Informationstechnologie mittels eines gezielten Förderprogramms vorangebracht werden muss. Ohne eine moderne IT-Infrastruktur wird Krankenhausversorgung in Zukunft nicht effizient leistbar sein. Wenn die Krankenhausstrukturen angepasst werden und Standorte wegfallen, ist es umso wichtiger, über telemedizinische Versorgungsangebote den unmittelbaren Anschluss an Fachexperten in allen Regionen sicherzustellen.

\subsection{Fazit}

Effiziente und qualitativ hochwertige Krankenhausversorgung erfordert stetige und ausreichend hohe Investitionen in Krankenhausstrukturen und Ausstattung. Wer eine klare Vorstellung davon hat, wie die Krankenhauslandschaft in Zukunft aussehen soll, kann auch notwendige Entscheidungen über den Investitionsbedarf und den richtigen Mitteleinsatz treffen. In Deutschland mangelt es nicht am Aufbau gesetzgeberischer Instrumente, um den Herausforderungen der Zukunft zu begegnen. Die Fülle an Regulierungsvorschriften und
Förderinstrumenten muss jedoch durchforstet, sinnvoll aufeinander abgestimmt und koordiniert weiterentwickelt werden. So ist es wichtig, über Qualitäts- und Strukturvorgaben auf der Bundesebene Maßstäbe für planerisches Handeln in den Ländern zu setzen. Diese rahmengebenden Vorgaben ersetzen aber keine zukunftsorientierte Bedarfsplanung.

Wenn alle für die Versorgung relevanten Akteure auf Landesebene zusammenarbeiten und gemeinsam ein Zielbild für Krankenhausstrukturen der Zukunft entwickeln, bleibt eine wohnortnahe und hochstehende medizinische Versorgung überall in Deutschland auch künftig realisierbar. Die Autoren schlagen vor, Krankenhausneubauten künftig durch Einzelförderung zu finanzieren. Krankenhausgebäude und medizinische Anlagen bringen eine Grundlast an Investitionsbedarf mit sich, die über leistungsorientierte Investitionspauschalen am besten refinanziert werden können. Der grundlegende Umbau der Krankenhausstrukturen kann weiterhin zielgerichtet über Fördermaßnahmen im Rahmen des Krankenhausstrukturfonds vorangebracht werden.

\section{Literatur}

Augurzky B, Beivers A, Emde A, Halbe B, Pilny A, Straub N, Wuckel C (2017) Stand und Weiterentwicklung der Investitionsförderung im Krankenhausbereich. Endbericht, Gutachten. Bundesministerium für Gesundheit, Essen, S 35

Augurzky B, Krolop S, Mensen A, Pilny A, Schmidt C, Wuckel C (2018) Krankenhaus Rating Report 2018, Personal - Krankenhäuser zwischen Wunsch und Wirklichkeit. Medhochzwei, Heidelberg, S 195

Bericht der Bundesregierung (1966) Bericht über die finanzielle Lage der Krankenanstalten. BT-Drucksache $\mathrm{V} / 4230$. Bundesregierung, Bonn, S 25

Busse R, Ganten D, Huster S, Reinhardt ER, Suttorp N, Wiesing U (2016) Zum Verhältnis von Medizin und Ökonomie im deutschen Gesundheitssystem - 8 Thesen zur Weiterentwicklung zum Wohle der Patienten und der Gesellschaft. https://www.leopoldina. org/uploads/tx_leopublication/Leo_Diskussion_ Medizin_und_Oekonomie_2016.pdf. Zugegriffen: 29. Aug. 2019

Deutscher Bundestag (1999) Gesetzentwurf der Fraktionen SPD und BÜNDNIS 90/DIE GRÜNEN. Entwurf 
eines Gesetzes zur Reform der gesetzlichen Krankenversicherung ab dem Jahr 2000 (GKVGesundheitsreform 2000). BT-Drucksache 14/1245. Deutscher Bundestag, Bonn

DKG (2018) Bestandsaufnahme zur Krankenhausplanung und Investitionsfinanzierung in den Bundesländern. Berlin, S. 73 und S. 109-110. https://www. dkgev.de/fileadmin/default/Mediapool/2_Themen/ 2.2_Finanzierung_und_Leistungskataloge/

2.2.2._Investitionsfinanzierung/2.2.2.1.

Investitionsfoerderung_der_Krankenhaeuser/

DKG_Bestandsaufnahme_KH-Planung_

Investitionsfinanzierung_2018_final.pdf. Zugegriffen: 1. Okt. 2019

DKG, PKV, GKV-SV (2019) Investitionsbedarf der Krankenhäuser: Aktuelle Auswertung bestätigt Unterfinanzierung durch die Bundesländer. Gemeinsame Presseerklärung 21.03.2019, Berlin. https:// www.gkv-spitzenverband.de/media/dokumente/ presse/pressemitteilungen/2018/Gm_2018-03-28_ Investitionsbewertungsrelationen.pdf. Zugegriffen: 1. Okt. 2019

endoCert (2018) Anforderungskatalog für EndoProthetikZentren der Maximalversorgung. https://doi.org/ 10.1055/B-9783131740816-00001. Zugegriffen: 1. Okt. 2019

Henriksen HE (2019) Digitalisierung in der Neuordnung des dänischen Krankenhausmarktes. In: Klauber J, Geraedts M, Friedrich J, Wasem J (Hrsg) Krankenhaus-Report 2019 - Das digitale Krankenhaus. Springer, Berlin Heidelberg, S 91

Hermann C (2007) Monistik ante portas - Notwendigkeiten und Wege des Umstiegs auf eine effizienzorientierte Krankhausfinanzierung. In: Klauber J, Robra BP, Schellschmidt H (Hrsg) Krankenhaus-Report 2006 - Krankenhaus im Umbruch. Schattauer, Stuttgart, S 101-116
InEK (2019a) G-DRG-report-browser 2019. https://www. g-drg.de/Datenbrowser_und_Begleitforschung/ G-DRG-Report-Browser/G-DRG-Report-Browser_ 2019. Zugegriffen: 1. Okt. 2019

InEK (2019b) Abschlussbericht, Entwicklung von Investitionsbewertungsrelationen (IBR) gem. § $10 \mathrm{KHG}$ für das Jahr 2019. InEK, Siegburg

Kanton Zürich Gesundheitsdirektion (2011) Zürcher Spitalplanung 2012, Strukturbericht, September 2011. https://gd.zh.ch/internet/gesundheitsdirektion/ de/themen/behoerden/spitalplanung_spitallisten/ akutsomatik.html. Zugegriffen: 1. Juli 2019

Loos S, Albrecht M, Zich K (2019) Zukunftsfähige Krankenhausversorgung - Simulation und Analyse einer Neustrukturierung der Krankenhausversorgung am Beispiel einer Versorgungsregion in NordrheinWestfalen. Bertelsmann Stiftung, Gütersloh https:// doi.org/10.11586/2019042

Mansky T, Drogan D, Nimptsch U, Günster C (2018) Eckdaten stationärer Versorgungsstrukturen für ausgewählte Behandlungsanlässe in Deutschland. In: Dormann F, Klauber J, Kuhlen R (Hrsg) Qualitätsmonitor 2018. Medizinisch Wissenschaftliche Verlagsgesellschaft, Berlin, S 210

Schelhase T (2019) Statistische Krankenhausdaten: Diagnosedaten der Krankenhauspatienten 2017. In: Klauber J, Geraedts M, Friedrich J, Wasem J (Hrsg) Krankenhaus-Report 2019. - Das digitale Krankenhaus. Springer, Berlin Heidelberg, S 275

Stephani V, Busse R, Geissler A (2019) Benchmarking der Krankenhaus-IT: Deutschland im internationalen Vergleich. In: Klauber J, Geraedts M, Friedrich J, Wasem J (Hrsg) Krankenhaus-Report 2019 - Das digitale Krankenhaus. Springer, Berlin Heidelberg, S 17

Tuschen KH, Trefz U (2010) Krankenhausentgeltgesetz. Kommentar, 2. Aufl. Kohlhammer, Stuttgart, S 54

Open Access Dieses Kapitel wird unter der Creative Commons Namensnennung 4.0 International Lizenz (http:// creativecommons.org/licenses/by/4.0/deed.de) veröffentlicht, welche die Nutzung, Vervielfältigung, Bearbeitung, Verbreitung und Wiedergabe in jeglichem Medium und Format erlaubt, sofern Sie den/die ursprünglichen Autor(en) und die Quelle ordnungsgemäß nennen, einen Link zur Creative Commons Lizenz beifügen und angeben, ob Änderungen vorgenommen wurden.

Die in diesem Kapitel enthaltenen Bilder und sonstiges Drittmaterial unterliegen ebenfalls der genannten Creative Commons Lizenz, sofern sich aus der Abbildungslegende nichts anderes ergibt. Sofern das betreffende Material nicht unter der genannten Creative Commons Lizenz steht und die betreffende Handlung nicht nach gesetzlichen Vorschriften erlaubt ist, ist für die oben aufgeführten Weiterverwendungen des Materials die Einwilligung des jeweiligen Rechteinhabers einzuholen. 